Präv Gesundheitsf 2006 1:140-144 DOI 10.1007/s11553-006-0021-5

Online publiziert: 14. März 2006

(c) Springer Medizin Verlag 2006
K. Meyer

Schweizerisches Gesundheitsobservatorium Neuchâtel \& Universität Bern

\title{
Körperliche Aktivität im Alter
}

\section{Mehr Differenzierung in Forschung und Praxis notwendig}

Die Lebenserwartung von Europäern und US-Amerikanern steigt derzeit um 3 Monate pro Jahr. Bis zum Jahr 2030 wird sich in Deutschland das Populationsverhältnis der über 6o-Jährigen zu den 20- bis 60Jährigen nahezu verdoppeln. Betrachtet man das Verhältnis der Altersgruppe der 80-Jährigen zu den 20- bis 6o-Jährigen, so wird es sich bis ins Jahr 2050 vervierfachen [1]. Für die Schweiz wird hochgerechnet, dass sich bis in das Jahr 2040 die Anzahl der Hochbetagten von 90 Jahren und älter verdoppeln, u. U. - bei einer weiteren deutlichen Zunahme der Lebenserwartung der Bevölkerung - sogar vervierfachen kann [2].

Vielfach sind mit dem Altern Gesundheitsprobleme, Behinderung und Abhängigkeit verbunden. Bei der Verzögerung der Entwicklung, der Abschwächung und/oder Kompensation dieser Probleme nimmt die regelmäßige körperliche $\mathrm{Be}$ wegung eine Schlüsselstellung ein. Prospektive Beobachtungsstudien auf Bevölkerungsebene zeigen, dass die körperlich aktiven Populationen einen besseren körperlichen Gesundheits- und Funktionszustand aufweisen als die inaktive Bevölkerung $[3,4]$.

Obschon noch viele Fragen zur Dosis-Wirkungs-Beziehung der körperlichen Aktivität mit älteren Menschen offen sind, ist heute belegt, dass ein Wechsel von sitzender Lebensweise zu einem aktiven Lebensstil - selbst wenn er erst im mittleren und älteren Lebensalter vorgenommen wird - die Mortalität reduzieren kann [3, 5]. In Querschnittstudien und prospektiven epidemiologischen Studien $[6,7]$ sowie experimentellen Studien [8] war regelmäßige körperliche Aktivität mit einer geringeren Prävalenz und Inzidenz von depressiven Symptomen sowie mit einem reduzierten Risiko einer kognitiven Verschlechterung assoziiert [9]. Positive Effekte der körperlichen Aktivität wurden auch bei Personen mit Multimorbidität wie z. B. kardiovaskulärer Erkrankung gezeigt [10], was die klinische Relevanz von körperlicher Bewegung unterstreicht. Viele dieser Erkenntnisse gehen auf randomisierte kontrollierte Studien zurück, in denen Ausdauer-, Kraftund Mobilitätstraining gezielt eingesetzt und deren Effekte belegt wurden [11].

\section{Bewegungsempfehlungen zu wenig differenziert}

Bestehende Bewegungsempfehlungen, die sich an die allgemeine Bevölkerung richten, sind überwiegend auf Sport und sportähnliche körperliche Aktivitäten begrenzt $[12,13]$. Das heißt, bislang wird unzureichend berücksichtigt, dass bei älteren und alten Menschen der Anteil nichtsportlicher körperlicher Aktivität einen relativ größeren Platz als sportliche Aktivität einnimmt. Obschon ausreichende Evidenz für die gesundheitliche Wirksamkeit von körperlicher Aktivität in der zweiten Lebenshälfte besteht, bleibt großer Bedarf an differenzierter Kenntnis über spezifische Formen und Methoden der körperlichen Aktivität und deren Wirkungen für spezifische Zielgruppen.

\section{Vielfalt der körperlichen Aktivität im Altersgang}

Gemäß allgemeiner Richtlinien für gesundheitsrelevante Bewegung weist in Deutschland, der Schweiz und den USA etwa zwei Drittel der älteren und alten Bevölkerung unzureichende körperliche Aktivität auf [14, 15, 16]. Dieser Kenntnisstand unterliegt möglicherweise einem Bias, der v. a. in der Definition von körperlicher Aktivität und der Definition der Gesundheitsrelevanz bei älteren Menschen verankert sein könnte.

Vielfalt körperlicher Aktivität. Im Leben älterer Menschen findet sich eine Vielfalt von körperlicher Bewegung, angefangen von regelrechter Sportaktivität wie Wandern, Gehen und Radfahren, über Gartenarbeit und muskulärer Aktivität, die beispielsweise bei der Bewältigung des Haushalts erbracht wird, bis hin zu Physiotherapie zur Kompensation von Gesundheitsproblemen und Behinderung. Auch für ausgewählte Gruppen älterer und alter Menschen, beispielsweise mit erhöhtem Sturzrisiko [17], Osteoporose [18] oder Sarkopenie [19] existieren spezifische Bewegungsprogramme. Auf Bevölkerungsebene jedoch führt die Synthese neuerer Literatur über gesundheitsrelevante Bewegungsempfehlungen zur Schlussfolgerung, dass der Kenntnisstand über optimale Methoden und Dosen der Aktivität zum Erreichen gesundheitsrelevanter Effekte sowie über minimale Aktivitätsschwellen für die Wirksamkeit un- 
zureichend ist [20]. In den berücksichtigten Studien wurden Personen der 6. und 7. Lebensdekade z. T. berücksichtigt, die der 8. Dekade und älteren Populationen blieben nahezu unberücksichtigt.

Erhebungsinstrumente. Eine Ursache für den unzureichenden Kenntnisstand könnte zum einen der Mangel an inadäquaten Instrumenten zur Erfassung der Vielfalt altersspezifischer körperlicher Bewegung sein. Existierende validierte Fragebögen für ältere Populationen sind eher selten zielgruppenspezifisch ausgerichtet $[21,22,23]$. Ferner erfassen die meisten der Instrumente die körperliche Bewegung als Verhalten [24, 25]; seltener wird der Energieverbrauch bei dieser Aktivität erfasst [26]. Zumindest für Populationen des jungen und mittleren Alters ist jedoch bekannt, dass weniger die Bewegung als solche, sondern der Energieverbrauch entscheidend ist, wenn es um die Prävention chronischer Erkrankungen geht [27]. Ob dieses Faktum gleichermaßen für ältere Menschen zutrifft, ist unklar.

\section{Klare Abgrenzung von Definitionen}

Neben den methodischen Lücken in Bezug auf die Erfassung der körperlichen Aktivität spielt auch die Definition des gesundheitlichen Outcomes eine zentrale Rolle, wenn es um die Erweiterung differenzierter Kenntnis über Bewegungseffekte im Alter geht. Die Zielvariable Gesundheit kann als objektive und/oder subjektive Gesundheit erfasst werden. Eine chronische Erkrankung kann in einer negativen Beziehung zur Prävalenz der körperlichen Bewegung stehen, dagegen kann eine gleichzeitige Erfassung und Analyse der wahrgenommenen Gesund- heit, Gesundheitseinstellung oder Selbstwirksamkeit diese negative Beziehung positiv modifizieren $[7,16,28]$.

Behinderung und funktionelle Einschränkungen werden $\mathrm{z}$. T. synonym erfasst, indem beispielsweise eine Verbesserung der Gehleistung durch Krafttraining mit einer Abschwächung der Schwere der Behinderung (zum Beispiel besseres Zurechtkommen mit Aktivitäten im Alltag) gleichgesetzt wird [29]. Eine klare Abgrenzung beider Begriffe ist eine Voraussetzung sowohl für die Entwicklung effizienter Interventionen als auch für die differenzierte Beurteilung von Interventionseffekten für das eine oder andere Problem.

\section{Hier steht eine Anzeige.}

\section{Springer}


Präv Gesundheitsf 2006 1:140-144 DOI 10.1007/s11553-006-0021-5

c) Springer Medizin Verlag 2006

\section{K. Meyer \\ Körperliche Aktivität im Alter. Mehr Differenzierung in Forschung und Praxis notwendig}

Zusammenfassung

Regelmäßige körperliche Aktivität nimmt eine Schlüsselstellung bei der Vermeidung bzw. Verzögerung altersbedingter Gesundheitsprobleme sowie bei der Kompensation deren Folgen ein. Bestehende allgemeine Empfehlungen für gesundheitsrelevante körperliche Bewegung sind überwiegend auf Sport und sportähnliche körperliche Aktivitäten begrenzt. Die bei älteren und alten Menschen häufiger vorkommende nichtsportliche körperliche Aktivität bleibt weitgehend unberücksichtigt. Obschon ausreichende Evidenz für die gesundheitliche Wirksamkeit von körperlicher Aktivität in der zweiten Lebenshälfte besteht, bleibt großer Bedarf an differenzierter Kenntnis über spezifische Formen und Methoden der körperlichen Aktivität und deren Wirkungen für spezifische Zielgruppen älterer und alter Men- schen. Gleiches trifft für spezifische Instrumente zur Erfassung der körperlichen Aktivität älterer und alter Menschen im weitesten Sinne zu.

Zukünftige Forschung sollte sich stärker auf die altersspezifischen Aspekte der körperlichen Aktivität sowie die Erfassung der Aktivität in der älteren und alten Bevölkerung ausrichten. Dieses Expertenstatement weist auf Forschungsfragen hin, deren Beantwortung zu einer Vereinheitlichung der Botschaft zur körperlichen Aktivität im Alter und zur Konsolidierung eines entsprechenden wissenschaftlichen und praktischen Konsenses beitragen kann.

Schlüsselwörter

Ältere Populationen · Körperliche Aktivität . Forschungsfragen

\section{Physical activity among the elderly. Greater differentiation in research and practice is needed}

\begin{abstract}
Regular physical activity is key to avoiding or delaying age-related health problems and minimising their impact. In Western societies, the old and older population (the 75+ population segment in particular) is the least likely to exercise or do the recommended amount of health-enhancing physical activity. Thus far, there has been insufficient understanding of and research on the prevalence, impact and effectiveness of the wide array of physical activities that the old and older population can do to improve their health and degree of independence. Along this line, nonsports-related activities should receive spe-
\end{abstract}

\section{Forschungsbedarf zur körperlichen Aktivität im Alter}

Es sind beträchtliche Lücken im Wissenstand über altersspezifisches Bewegungsverhalten sowie alterspezifisch adäquate körperliche Aktivitäten, die mit gesundheitlich positiven Wirkungen verbunden sind, zu füllen. Dringender Forschungsbedarf ergibt sich v. a. in den Bereichen - spezifischer Erhebungsmethoden für körperliche Aktivität und

- zielgruppenspezifischer Methoden der körperlichen Aktivität und ihrer Effekte.

\section{Methoden der Aktivitätserfassung und Aktivitätszuweisung}

Die Weiterentwicklung von Erhebungsmethoden, die die Vielfalt und Spezifika der körperlichen Aktivität im Alter sowie deren Energieverbrauch differenziert erfassen, ist eine unverzichtbare Basis für weitere physiologische, psychosozial- und strukturfokussierte Forschungsfragen sowie für praktische Ansätze der Bewegungsförderung im Alter. In solchen Instrumenten ist auch die sich im Lebensverlauf verändernde Wahrnehmung der Begriffe von Sportaktivität und körperlicher Aktivität zu beachten. Ferner sind in Bezug auf die Prävalenzbestimmung der körperlichen Aktivität Variablen zu erfassen, die in der statistischen Analyse eine Differenzierung von Alters -, Kohortenund Periodeneffekten ermöglichen.

Die Heterogenität und Mulitmorbidität älterer Populationen verlangt eine entsprechende Verordnung von bzw. Zuweisung zu Bewegungsprogrammen. Dies impliziert die Notwendigkeit der Entwicklung eines evidenzbasierten Procederes (zum Beispiel Aktivitätsfragebogen; motorische Funktionstests; Get-up-andgo-Test; kardiopulmonale Belastungstests), mit dem ältere und alte Menschen für individuell adäquate Bewegungsinterventionen identifiziert werden können (Triage).

\section{Bewegungsmethoden und Effekte}

In welcher Weise sind übliche Bewegungsinterventionen (Ausdauer-, Kraft- und Koordinationstraining) für Subgruppen 
älterer und alter Menschen, z. B. mit chronischen Krankheiten/körperlichen Behinderungen, kognitiven Einschränkungen, depressiven Störungen sowie für Hochbetagte, sozial isolierte Ältere und städtisch vs. ländlich lebende Populationen) spezifisch zu modifizieren?

Eine Vielzahl randomisierter kontrollierter Studien belegt, dass ein definiertes Ausdauertraining oder Krafttraining u. a. der Kraftverbesserung, der Sturzprophylaxe und der besseren Bewältigung des praktischen Alltags älterer Menschen dient. Es fehlt jedoch an empirischer und experimenteller Evidenz darüber, ob und in welcher Weise die Vielfalt der nichtsportlichen körperlichen Bewegung positive Effekte auf definierte Outcomegrößen zur Leistungsfähigkeit und körperlichen Funktionsfähigkeit haben. Bei der Beantwortung dieser Frage sind pathophysiologische, psychisch-mentale und soziale Ausgangssituationen der zu beobachtenden Personen zu berücksichtigen.
Bislang ist unklar, ob ein gegebener Bewegungsreiz bei einem älteren Menschen zu vergleichbaren Anpassungen führt wie bei einem jüngeren Menschen. Dies erfordert die Erarbeitung einer präziseren Dosis-Wirkungs-Abhängigkeit für ältere und alte Menschen. Hierbei ist die Heterogenität der älteren Bevölkerung zu berücksichtigen.

Von großer Wichtigkeit für den Erfolg einer Bewegungsförderung im Alter ist auch die Beantwortung der Frage, welchen Einfluss zurückliegende Bewegungserfahrungen auf das gegenwärtige Bewegungsverhalten haben und wie sie für das zukünftige Bewegungsverhalten genutzt werden können. Dies impliziert eine verstärkte Erforschung des Phänomens der Selbstwirksamkeit in älteren Populationen.

Es sind spezifische kognitiv-verhaltensorientierte Interventionsstrategien $\mathrm{zu}$ entwickeln, die eine Verbesserung der Compliance für langfristige körperliche
Aktivität bewirken. Dies ist insbesondere für Populationen mit chronischen körperlichen Problemen, depressiven Störungen und kognitiven Einschränkungen, deren Folgen durch regelmäßige körperliche Aktivität positiv beeinflusst werden können, von Bedeutung.

\section{Politisch relevante Fragen}

Neben den Fragestellungen zu Methoden und Wirkungen der körperlichen Aktivität sollte eine systematische Bestandsaufnahme des Engagements zum einen auf der Policy-Ebene, zum anderen auf der Ebene „Umwelt und Städteplanung“ Handlungsbereiche und Handlungsansätze aufdecken, über die der Zugang zu Bewegungsangeboten für die ältere Bevölkerung verbessert werden kann.

Die ökonomische Evaluation von körperlicher Aktivität bzw. von Bewegungsmangel kann dazu beitragen, die Politik auf das Thema „Bewegungsförderung im

\section{Hier steht eine Anzeige.}


Alter" aufmerksam zu machen und nachfolgende legislative und exekutive Konsequenzen anzustoßen.

\section{Fazit für die Praxis}

Die Ergebnisse einer stärker altersorientierten Forschung zur körperlichen Aktivität:

fördern eine einheitliche Botschaft
und Konsolidierung des wissen-
schaftlichen Konsenses über adä-
quate Methoden der körperlichen
Bewegung und ihre Erfassung in
spezifischen Zielgruppen,
liefern Hinweise und Evidenz für ei-
ne altersspezifische Modifikation
der existierenden Richtlinien zur ge-
sundheitsrelevanten körperlichen
Aktivität und
erhöhen die Effizienz in Bezug auf
Verordnung und Anwendung der
körperlichen Aktivität unter Berück-
sichtigung seiner altersspezifischen
Variabilität.

Ferner tragen die Ergebnisse zur Verbesserung von strukturellen Bedingungen bei, die der älteren und alten Bevölkerung einen flächendeckenden Zugang zu mehr altersspezifischer körperlicher Aktivität ermöglichen.

\section{Korrespondierender Autor}

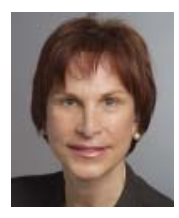
PD Dr. K. Meyer

Schweizerisches Gesundheitsobservatorium Neuchâtel \& Universität Bern Espace de l'Europe, 2010 Neuchâte

meyer.katharina@bluewin.ch

Interessenkonflikt. Es besteht kein Interessenkonflikt. Der korrespondierende Autor versichert, dass keine Verbindungen mit einer Firma, deren Produkt in dem Artikel genannt ist, oder einer Firma, die ein Konkurrenzprodukt vertreibt, bestehen. Die Präsentation des Themas ist unabhängig und die Darstellung der Inhalte produktneutral.

\section{Literatur}

1. Birg H (2001) Die demographische Zeitenwende. Der Bevölkerungsrückgang in Deutschland und Europa. Beck, München

2. Bundesamt für Statistik (2004) Statistik. Bundesamt für Statistik, Neuenburg, Schweiz
3. Blair SN, Kohl H, Barlow C, Paffenbarger RS, Gibbons L, Mnceras C (1995) Changes in physical fitness and all-cause mortality: a prospective study of healthy and unhealthy mean. JAMA 273: 10931098

4. Ratanen T, Guralnik J, Sakari-Rantala R et al. (1999) Disability, physical activity, and muscle strength in older women: the Women's Health and Aging Study. Arch Phys Med Rehabil 80: 130-135

5. Bijnen F, Feskens E, Caspersen C (1999) Baseline and previous physical activity in relation to mortality in elderly men: the Zutphen Elderly Study. Am J Epidemiol 150: 1621-1628

6. Hughes JR (1984) Psychological effects of habitual aerobic exercise: a critical review. Prev Med 13: 6678

7. McAuley E, Rudolph D (1995) Physical activity, aging, and psychological well-being. J Phys Act Aging 3: 67-96

8. Blumenthal J, Babyak M, Moore K et al. (1999) Effects of exercise training on older patients with major depression. Arch Intern Med 159: 23492356

9. Laurin D, Verrault R, Linday J, Mac Pherson K, Rockwood K (2001) Physical activity and risk of cognitive impairment and dementia in elderly persons. Arch Neurol 58: 498-504

10. Hu F, Stampfer M, Colditz G (2000) Physical activity and risk of stroke in women. JAMA 182:2961-2967

11. King AC, Rejeski WJ, Buchner DM (1998) Physical activity interventions targeting older adults: A critical review and recommendations. Am J Prev Med 15: 316-333

12. American College of Sports Medicine (2006) ACSM's Guidelines for Exercise Testing and Prescription, 7th edn. American College of Sports Medicine, Philadelphia

13. Pate RR, Pratt M, Blair SN et al. (1995) Physical activity and public health: a recommendation from the Centers for Disease Control and Prevention and the American College of Sports Medicine. JAMA 273: 402-407

14. U.S. Department of Health and Human Services (1996) Physical activity and health: a report of the Surgeon General. Department of Health and Human Services, Centers for Disease Control and Prevention, National Center for Chronic Disease Prevention and Health Promotion, Atlanta, Georgia, USA

15. Breuer C (2005) Cohort effects in physical activity. Public Health 13: 189-195

16. Meyer K (2005) Physical activity of adults aged 50 years and older in Switzerland. Soz Präventivmed 50: 218-229

17. Robertson M, Devlin N, Gardner M, Campbell A (2001) Effectiveness and economic evaluation of a nurse delivered home exercise program to prevent falls. Randomized controlled trial. Br Med J 322: 16

18. Bloomfield SA, Smith SS (2003) Osteporosis. In: Durstine JL, Moore GE (eds) ACSM's exercise management for persons with chronic diseases and disabilities, 2nd edn. Human Kinetics, Champaign, IL, pp 222-229

19. Evans WJ (1996) Reversing sarcopenia: How weight training can built strength and vitality. Geriatrics 51: 46-53

20. Bouchard C (2001) Physical activity and health: Introduction to the dose-response symposium. Med Sci Sports Excer 33: 345-369

\section{September 2006}

Leipzig 21.-23.09.2006

Gemeinsamer Kongress der DGMP und DGMS

Medizintechnischer und soziodemographischer Wandel- Herausforderungen an die psychosoziale Medizin

Auskunft: Herr Prof. Dr. Elmar Brähler

Selbstständige Abteilung für Medizinische Psychologie und Medizinische Soziologie der Universität Leipzig, Philipp-Rosenthal-Str. 55, 04103 Leipzig,

Fon: 0341-9718803

Fax: 0341-9718809,

www.dgmp-dgms-2006-uni-leipzig.de/

\section{November 2006}

Dresden, Deutsches Hygiene-Museum 30.11.02.12.2006, DVG PH

Professionalisierung in der Öffentlichen Gesundheit für Südosteuropa: Heute und Morgen

1. Konferenz der Dekane und Direktoren der Schools of Public Health und der Präsidenten der Nationalen Gesellschaften für Öffentliche Gesundheit in Südosteuropa

Auskunft: Herr Prof. Dr. Dr. W. Kirch,

Forschungsverbund Public Health Sachsen und Sachsen-Anhalt, Fiedlerstr. 33, 01307 Dresden,

Fon: 03514584490

Fax: 03514585338 ,

public.health@mailbox.tu-dresden.de 